6. Chapman PB, et al. Improved survival with vemurafenib in melanoma with BRAF V600E mutation. NEngl J Med. 2011;364(26):2507-2516.

7. Lee S-Y, et al. FAM83A confers EGFR-TKI resistance in breast cancer cells and in mice. J Clin Invest. 2012;122(9):3211-3220.

8. Cipriano R, et al. FAM83B mediates EGFR- and RAS-driven oncogenic transformation. J Clin Invest. 2012;122(9):3197-3210.

9. Avraham R, Yarden Y. Feedback regulation of EGFR signalling: decision making by early and delayed loops. Nat Rev Mol Cell Biol. 2011;12(2):104-117.

10. She QB, Solit D, Basso A, Moasser MM. Resistance to gefitinib in PTEN-null HER-overexpressing tumor cells can be overcome through restoration of PTEN function or pharmacologic modulation of constitu- tive phosphatidylinositol 3'-kinase/Akt pathway signaling. Clin Cancer Res. 2003;9(12):4340-4346.

11. Normanno N, et al. The MEK/MAPK pathway is involved in the resistance of breast cancer cells to the EGFR tyrosine kinase inhibitor gefitinib. Cell Physiol. 2006;207(2):420-427.

12. Ng KP, et al. A common BIM deletion polymorphism mediates intrinsic resistance and inferior responses to tyrosine kinase inhibitors in cancer. Nat Med. 2012;18(4):521-528.

13. Qi XJ, Wildey GM, Howe PH. Evidence that Ser87 of BimEL is phosphorylated by Akt and regulates BimEL apoptotic function. J Biol Chem. 2006;281(2):813-823.

14. Ley R, Ewings KE, Hadfield K, Howes E, Balmanno $\mathrm{K}$, Cook SJ. Extracellular signal-regulated kinases
$1 / 2$ are serum-stimulated " $\mathrm{Bim}(\mathrm{EL})$ kinases" that bind to the BH3-only protein Bim(EL) causing its phosphorylation and turnover. $J$ Biol Chem. 2004;279(10):8837-8847.

15. Harada H, Quearry B, Ruiz-Vela A, Korsmeyer SJ. Survival factor-induced extracellular signal-regulated kinase phosphorylates BIM, inhibiting its association with BAX and proapoptotic activity. Proc Natl Acad Sci U S A. 2004;101(43):15313-15317.

16. Ewings KE, et al. ERK1/2-dependent phosphorylation of $\operatorname{Bim}(\mathrm{EL})$ promotes its rapid dissociation from Mcl-1 and $\mathrm{Bcl}-\mathrm{x}(\mathrm{L}) . E M B O J .2007$; 26(12):2856-2867.

17. Kolch W, Pitt A. Functional proteomics to dissect tyrosine kinase signalling pathways in cancer. Nat Rev Cancer. 2010;10(9):618-629.

\title{
Mining the secrets of the CSF: developing biomarkers of neurodegeneration
}

\author{
William Z. Potter
}

National Institute of Mental Health, Bethesda, Maryland, USA.

\begin{abstract}
Our ability to track the progression of neurological disorders like Parkinson's disease (PD) is hampered by a lack of biomarkers, rendering the neuronal changes that underlie clinical symptoms largely a mystery. In this issue of the JCI, Fanara et al. report the development of an innovative approach to biomarker development. They describe a method to measure axonal microtubule function via cerebrospinal fluid (CSF) sampling and use this technique to provide evidence of deficiencies in this process in PD patients. This both sheds light on the pathophysiology of PD and has implications for the more general problem of developing biomarkers for any brain process.
\end{abstract}

\section{The problem}

The underlying pathologic processes driving the symptoms of neurodegenerative disease cannot be followed in living subjects without better biomarkers. The difficulty in identifying such biomarkers of disease progression has impeded the development of therapeutic strategies to combat it. The study by Fanara et al. on cerebrospinal fluid (CSF) kinetic biomarkers of axonal transport in this issue of the $J C I$ marks an important contribution to the field of measuring difficult-to-assess brain processes in vivo (1). The authors' work expands on a series of earlier studies in murine models that used heavy water $\left({ }^{2} \mathrm{H}_{2} \mathrm{O}\right)$ labeling to investigate microtubule dynamics and stabilization by taxanes with neuroprotective potential $(2,3)$. Here,

Conflict of interest: William Z. Potter holds stock in Lilly and Merck and has served as a consultant for Envoy.

Citation for this article: J Clin Invest. 2012; 122(9):3051-3053. doi:10.1172/JCI65309.
Fanara et al. systematically developed a biomarker of microtubule function (1), and their work has implications for the more general problem of developing biomarkers for any brain process.

Ideally, in the development of biomarkers intended for human application, one needs a model system in which variables can be controlled and manipulated. Although genetic or lesion models of human disease are available, to be clinically relevant, the direct measures possible in animal brain coupled to a biomarker analysis must be translatable to humans. CSF is an attractive sample source. Although technical issues involved in sampling from mice have discouraged many groups, Fanara et al. provide a compelling case that it is possible to relate a pathologic process in a murine model to a specific CSF analyte measurable in mice and humans.

\section{CSF as a biomarker source}

To appreciate the potential importance of a wider application of this strategy of bio- marker development, we must consider what traditional human CSF studies have and have not been able to deliver in the past. Human CSF has been a source of biomarkers, especially as an index of drug action, for decades. For instance, antidepressants were shown to produce hypothesized effects on CSF amine neurotransmitter metabolites in the early 1970 s (4). The kinetic principles for interpreting concentrations of analytes in CSF were established during this same period, including the use of stable isotopes (5). Recently, especially in Alzheimer's disease (AD), discovery proteomic assays have identified a number of analytes beyond amyloid- $\beta$ and phospho-tau, increasing interest in CSF as a source of biomarkers of disease (6). Even when robust relationships can be established between a CSF analyte and a disease process or drug action, gaps remain in our knowledge regarding how best to interpret a concentration change. Definitive interpretations require more precise knowledge of the sites of formation of the analyte as well as the processes that control its concentration in CSF.

Figure 1 provides a minimal model of the processes that would need to be quantitatively understood in order to fully interpret behavior of any CSF analyte. Even with simplifying assumptions, such as common rate constants for molecule formation and movement from all brain tissues into the CSF, other rate constants are relevant to our understanding; these include not only direct egress into peripheral compart- 


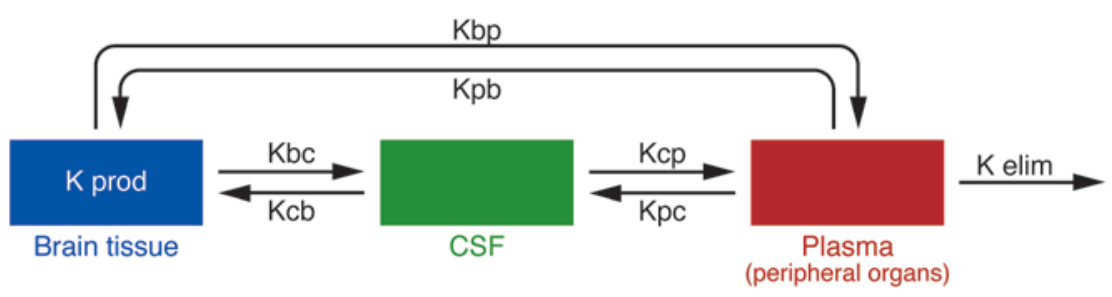

Figure 1

Model of equilibrium processes for any analyte in CSF. The level of any analyte in the CSF is related to the rate of production (K prod), the rate of egress into the CSF $(\mathrm{Kbc})$, the rate of entry into brain tissue from the CSF $(\mathrm{Kcb})$, the rate of entry into the periphery from the CSF $(\mathrm{Kcp})$, the rate of entry into the CSF from plasma $(\mathrm{Kpc})$, the rate of egress from brain to plasma (Kbp), the rate of entry into the brain from plasma $(\mathrm{Kpb})$, and the rate of elimination from the plasma ( $\mathrm{K}$ elim). The model is simplified: rates include all processes, whether passive (diffusion) or active (release, uptake, or transport depending on tissue and direction), and sites of production are treated as homogenously distributed through brain tissue.

ments, but any reentry into brain tissue or CSF, as well as the contribution of peripheral production. Since very large concentration gradients (up to 10,000-fold) of analytes are observed between blood and CSF, with the direction depending on the analyte, the concentration in one compartment has the potential to exert a substantial influence on the other. Thus, a peripheral sink hypothesis has been invoked to argue that simply binding amyloid- $\beta$ in blood with a monoclonal antibody may deplete brain concentrations in $\mathrm{AD}(7)$, although the rate constants to support this hypothesis have not been ascertained. Such concerns are of more than academic interest, given their relevance to selecting optimal doses for clinical studies. In the case of monoclonal antibodies for $\mathrm{AD}$ therapy, concentrations in brain - rather than only in blood - may prove to be the basis of CNS effects. The interpretation of negative results from a just-completed phase III trial of the monoclonal antibody bapineuzumab (8) may require additional studies to address the unknown rate constants driving movement of the antibody in and out of the brain, as well as how it binds to and clears amyloid- $\beta$.

\section{New applications to studies of human disease}

In order to be more confident of our interpretation of the source of alterations in any CSF analyte, we could initiate a variety of studies in animals and humans to address the gaps in our knowledge on the various rate constants shown in Figure 1. Alternatively, one could radiolabel precursors of prespecified analytes of interest and track a specific structure or process. Fanara et al. used this alternative approach to investigate how selected deuterated cargo proteins move from the site of synthesis to the site of release via microtubule-mediated axonal transport (1). Briefly, they established in murine models of neurodegeneration that the rate of appearance of selected deuterated proteins was delayed in proportion to microtubule disruption, then inferred evidence of altered microtubule disruption in patients with Parkinsonism who showed an analogous delay in appearance of the same proteins after loading with ${ }^{2} \mathrm{H}_{2} \mathrm{O}$. Similarly, a stable isotope precursor amino acid, ${ }^{13} \mathrm{C}$-leucine, has been used in another context to show that a $\gamma$-secretase inhibitor has a larger effect on the formation of new amyloid- $\beta$ in the CSF than would be concluded if one measured only the total unlabeled pool, a finding used to justify doses of that inhibitor for a trial in $\mathrm{AD}(9)$.

In the Fanara et al. study, the interpretation of the human data requires that the study of the animal model, in which abnormal microtubule altered rates of biomarker appearance, is translatable to humans (1). Prior to these studies, there was no reasonable way to link alterations in any analyte that could be measured in CSF or any other fluid tissue compartment (e.g., blood and urine) to abnormal microtubule function. There remains the possibility that some analyte or combination of analytes could ultimately also be linked to abnormal microtubule function, either through correlation with the values generated by the ${ }^{2} \mathrm{H}_{2} \mathrm{O}$ approach or by another series of preclinical studies. But the likelihood that the same specific analytes and determinants of their rates of formation and clearance would have an analogous relationship to microtubule function across species seems low. The rate of ${ }^{2} \mathrm{H}_{2} \mathrm{O}$-labeled appearance in the CSF of a specific analyte that can be firmly linked to a specific process in preclinical models and then given some validation in the related human disease may offer a much higher probability of identifying a useful biomarker of a specific process, as the authors propose.

\section{Unanswered questions}

Some cautions are, however, in order as to the relative value of this approach versus comprehensive proteomic and metabolomic studies. Aside from purely quantitative technical issues related to incorporation of ${ }^{2} \mathrm{H}_{2} \mathrm{O}$ into any particular analyte, there are the more fundamental questions of rates of incorporation and elimination which require - at a minimum - calculation of the area under the curve (AUC) of concentration versus time. Fanara et al. rely on a global measure of protein elimination from CSF to rule out effects on that process (1), which could miss effects on analytes that constitute a small fraction of total protein as well as leave open the possibility that the unexplored determinants of CSF concentration (Figure 1) have some influence. In the one patient the authors describe in whom multiple CSF measures were available, visual inspection suggested that not only was the peak time of labeled cargo protein delayed, but also there was an increase in the AUC (1). If real, this could reflect an increased rate of production as well as decreased efficiency of microtubule transport and/or a quicker, earlier removal from CSF (e.g., leakage due to injury) that is saturated, leading to a later peak. Neuronal injury might affect the rate of change in the CSF concentration of any analyte released from that neuron. The direction of change is not easy to predict, since damage could increase reentry rate through leakage or decrease it if an active process were involved. Rates of formation of neuronal products may be altered as well. In animal models, of course, one can rule out changes in formation rates, as was done in the present study of toxin effects on microtubule-mediated transport (1). But in an actual human disease, without a measure of rates of formation and removal from CSF as well as rate of appearance in that pool, the question of the major determinants of any alteration remains open.

\section{Conclusions}

Independent of these caveats, as Fanara et al. make clear, it could be that an altera- 
tion of the rate of appearance of one or another specific cargo proteins in CSF after ${ }^{2} \mathrm{H}_{2} \mathrm{O}$ labeling could reveal neuronspecific defects that occur early in a disease process. More generally, stable isotope labeling of precursor pools followed by either cross-sectional or longitudinal CSF sampling offers a powerful tool to explore aspects of pathologic processes in the brain that are unlikely to be detected by either proteomic or metabolomic measures of absolute steady-state concentrations. Combining measures of total analyte and rates of appearance of labeled newly synthesized forms should increase our ability to define early stages of neurodegenerative diseases and facilitate the development and testing of novel therapies targeted to the pathologic process. In other words, treating the biomarker (e.g., cholesterol) may offer a path to prevent- ing or delaying the clinical symptoms that lie downstream of that biomarker and are often irreversible consequences of failing to intervene early.

\section{Acknowledgments}

The author thanks Julie Stone (Merck Research Laboratories) for sharing her insights on what needs to be understood to fully interpret changes in CSF concentrations of analytes.

Address correspondence to: William Z. Potter, NIMH, 6001 Executive Blvd., BG NSC RM 7209, Rockville, Maryland 20892, USA. Phone: 267.970.6604; E-mail: wzpottermd@gmail.com.

1. Fanara $P$, et al. Cerebrospinal fluid-based kinetic biomarkers of axonal transport in monitoring neurodegeneration. J Clin Invest. 2012; 122(9):3159-3169.
2. Fanara $P$, et al. In vivo measurement of microtubule dynamics using stable isotope labeling with heavy water. Effect of taxanes. J Biol Chem. 2004; 279(48):49940-49947.

3. Fanara P, et al. Stabilization of hyperdynamic microtubule is neuroprotective in ALS.J Biol Chem. 2007;282(32):23465-23472

4. Bertilsson L, Asberg M, Thoren P. Differential effect of chlorimipramine and nortriptyline on cerebrospinal fluid metabolites of serotonin and noradrenaline in depression. Eur J Clin Pharmacol. 1974; 7(5):365-368

5. Kopin IJ. Measuring turnover of neurotransmitters in human brain. In: Lipton MA, DiMascio A, Killam KF, eds. Psychopharmacology: A Generation of Progress. New York, New York, USA: Raven Press; 1978:933-942.

6. Hu WT, et al. Novel CSF biomarkers of Alzheimer's disease and mild cognitive impairment. Acta Neuropathol. 2010;119(6):669-678.

7. Zlokovic BV. Clearing amyloid through the bloodbrain barrier. J Neurochem. 2004;89(4):807-811.

8. Pollack A. Alzheimer's drug fails its first big clinical trial. New York Times. July 24, 2012:B4.

9. Bateman RJ, et al. A $\gamma$-secretase inhibitor decreases amyloid- $\beta$ production in the central nervous system. Ann Neurol. 2009;66(1):48-54

\title{
When NK cells overcome their lack of education
}

\author{
Baptiste N. Jaeger ${ }^{1,2,3}$ and Eric Vivier ${ }^{1,2,3,4}$
}

${ }^{1}$ Centre d'Immunologie de Marseille-Luminy, Aix-Marseille Université UM2, Campus de Luminy case 906, Marseille, France. ${ }^{2}$ INSERM U1104, Marseille, France. ${ }^{3}$ CNRS, UMR7280, Marseille, France. ${ }^{4}$ Assistance Publique — Hôpitaux de Marseille, Hôpital de la Conception, Marseille, France.

\begin{abstract}
Cells of the immune system have evolved various molecular mechanisms to sense their environment and react to alterations of self. NK cells are lymphocytes with effector and regulatory functions, which are remarkably adaptable to changes in self. In a study published in this issue of the JCI, Tarek and colleagues report the clinical benefits of manipulating NK cell adaptation to self in an innovative $\mathrm{mAb}$-based therapy against neuroblastoma (NB). This novel therapeutic strategy should stimulate further research on NK cell therapies.
\end{abstract}

NK cells are involved in the elimination of tumor cells and infected cells (1); they can kill their cellular targets via cytotoxic granule exocytosis and also secrete cytokines such as IFN- $\gamma$ that participate in the shaping of the adaptive immune response. NK cells express a wide range of surface molecules that include inhibitory and activating receptors; in humans, this family comprises the natural cytotoxicity receptors (NKp30, NKp44, and NKp46), the Fcy receptor IIIA (CD16), and the activating killer cell immunoglobulin-like receptors (KIRs). CD16 endows NK cells with antibody-dependent cell-mediated cytotoxicity

Conflict of interest: Eric Vivier is a cofounder and shareholder of Innate Pharma.

Citation for this article: J Clin Invest. 2012; 122(9):3053-3056. doi:10.1172/JCI63524.
(ADCC) properties. These activating receptors associate with immunoreceptor tyrosine-based activation motif-bearing adaptors to transduce potent activating signals. The inhibitory KIRs in humans (and their functional homologs in mice, the Ly49 receptors) recognize classical major histocompatibility complex class Ia molecules (MHC-I) and transduce inhibitory signals via their intracytoplasmic tyrosine-based inhibition motifs. Upon NK cell encounter with potential target cells, the integration of activating and inhibitory signals dictates the NK cell response. Normal self cells expressing high levels of MHC-I and low levels of activating ligands are spared, while stressed cells with downregulated MHC-I expression and high levels of activating ligands activate NK cells and are killed.

\section{NK cell education}

The functional maturation of NK cells includes a process of education, also referred as to licensing, arming, or tuning, by which NK cells acquire effector functions that are adapted to the host in which they develop (2-5). In addition to its wellknown role in the regulation of NK cell effector functions, MHC-I recognition by inhibitory receptors is also involved in NK cell education. When NK cells cannot sense self MHC-I, as in MHC-I-deficient mice or patients, individuals do not develop autoimmune disorders, and the NK cells are hyporesponsive to stimulation in vitro (Figure 1A) (6-8). Various conflicting models have been proposed to describe how the interaction of MHC-Ispecific receptors with their ligands contributes to NK cell education (2-5). A unifying model inspired from the arming/ disarming model initially proposed by David Raulet and colleagues (2) is consistent with the experimental data published so far. In this scenario, NK cells sense target cells via the combined engagement of activating, inhibitory, and adhesion receptors. The intensity of the NK cell response is commensurate with the inte- 